\title{
Optimization of the optical array geometry for
} IceCube-Gen2

\section{The IceCube-Gen2 Collaboration}

(a complete list of authors can be found at the end of the proceedings)

E-mail: anastasiia.yovych@icecube.wisc.edu

IceCube-Gen2 is a planned extension of the IceCube Neutrino Observatory at the South Pole designed to study the high-energy neutrino sky from $\mathrm{TeV}$ to $\mathrm{EeV}$ energies with a five times better point source sensitivity than the current IceCube detector. This is achieved by deploying 120 new strings with attached optical sensors in a pattern around IceCube that features considerably larger distances between individual strings than the $\sim 125 \mathrm{~m}$ for the existing detector. Here, we present the results of an optimization study searching for the best point source sensitivity while varying the IceCube-Gen2 string spacing between $150 \mathrm{~m}$ and $350 \mathrm{~m}$.

Corresponding author: Anastasiia Omeliukh ${ }^{a, *}$

a DESY, Platanenallee 6, 15738 Zeuthen, Germany

${ }^{*}$ Presenter

$37^{\text {th }}$ International Cosmic Ray Conference (ICRC 2021)

July 12th-23rd, 2021

Online - Berlin, Germany 


\section{IceCube-Gen2}

IceCube is a cubic-kilometer neutrino detector installed in the ice at the geographic South Pole [1] between depths of $1450 \mathrm{~m}$ and $2450 \mathrm{~m}$, completed in 2010. Reconstruction of the direction, energy and flavor of the neutrinos relies on the optical detection of Cherenkov radiation induced by charged particles produced in the interactions of neutrinos in the surrounding ice or the nearby bedrock.

IceCube-Gen2 will be an extension of the existing IceCube [2] array aiming to explore the highenergy neutrino sky. 120 new strings with attached optical sensors will be installed around IceCube with considerably larger distances between individual strings, increasing the sensitivity for neutrino energies above $10 \mathrm{TeV}$. A large surface array will enhance the veto of cosmic-ray (CR) air showers and, thus, lower the energy threshold for the identification of neutrino interactions on the Southern sky. The detection of neutrinos with energies above $100 \mathrm{PeV}$ is the main goal of the radio array [3]. As a first step towards IceCube-Gen2, IceCube Upgrade [4], currently under construction, lowers the detection threshold for neutrinos to $1 \mathrm{GeV}$ leading to significant improvements for oscillation measurements, dark matter, and other beyond Standard Model physics searches. Here we focus on the optimization of the inter-string spacing for best point source sensitivity of the high-energy optical array that is comprised of the 86 IceCube and 120 additional strings.

The IceCube-Gen2 optical array will, like IceCube, detect neutrinos via the relativistic products of charge current (CC) and neutral current (NC) interactions. Muons created in $v_{\mu}$ CC interactions produce tracks when passing through the detector. Cascades are produced for all $v_{e}$ and $v_{\tau}$, as well as $v_{\mu} \mathrm{NC}$ interactions. In this work, we study the sensitivity to point sources with tracks.

\section{2. “Sunflower" geometries}

A previous study of geometrical shapes of the IceCube-Gen2 optical array [5] has shown that a "Sunflower"-like geometry is advantageous compared to IceCube's regular grid. The location of the strings in "Sunflower" geometry is defined in a polar coordinate system as

$$
r=s \sqrt{n}, \quad \phi=\frac{2 \pi}{g^{2}} n
$$

where $g=\frac{1+\sqrt{5}}{2}$ (golden ratio), $s$ is a spacing parameter, and $n$ is a natural number that defines string position. To generate the "Sunflower" geometry, the spiral starts at the center of IceCube. All the string positions that are inside of IceCube region or outside of the allowed (cf. Fig. 1) construction region are removed. The process is stopped when the total number of 120 strings is reached.

The IceCube-Gen2 optical sensors will be placed between $1325 \mathrm{~m}$ and $2575 \mathrm{~m}$ underground with $16 \mathrm{~m}$ spacing between the DOMs along the string. Eight values of inter-string spacing parameter $s$ were chosen for the optimization study: $150 \mathrm{~m}, 200 \mathrm{~m}, 220 \mathrm{~m}, 240 \mathrm{~m}, 260 \mathrm{~m}, 280 \mathrm{~m}$, $300 \mathrm{~m}$, and $350 \mathrm{~m}$. Fig. 1 shows the schematic top view of the high-energy optical array layout for three of these parameters $(150 \mathrm{~m}, 240 \mathrm{~m}$, and $350 \mathrm{~m})$. 


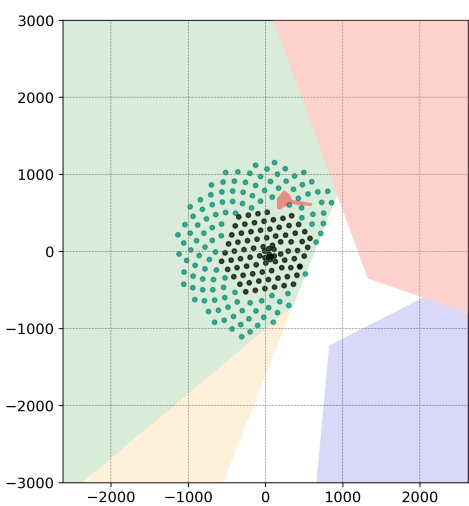

(a) $\mathrm{s}=150 \mathrm{~m}$

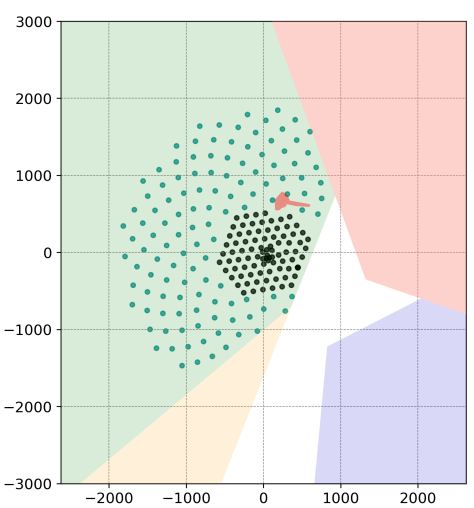

(b) $\mathrm{s}=240 \mathrm{~m}$

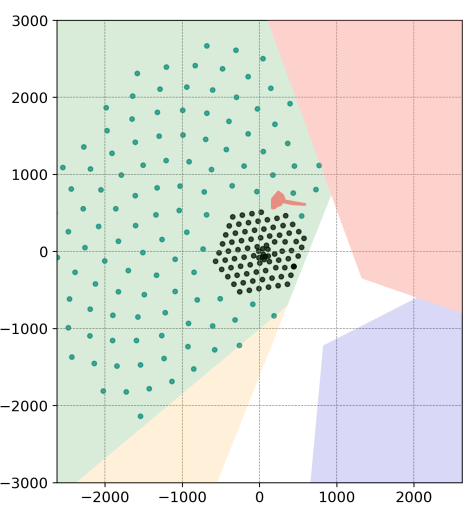

(c) $\mathrm{s}=350 \mathrm{~m}$

Figure 1: Visualization of IceCube-Gen2 geometries with inter-string spacings of $150 \mathrm{~m}$ (a), $240 \mathrm{~m}$ (b), and $350 \mathrm{~m}(\mathrm{c})$. The black dots represent the location of existing IceCube strings. The small red region near the IceCube detector is an old South Pole station and unsuitable for deployment. The allowed region for IceCube-Gen2 construction is limited to the light-green sector. All other sectors are reserved for different purposes.

\section{Monte Carlo simulations}

IceCube-Gen2 will have novel optical sensors such as mDOMs and D-Eggs [2]. The multiPMT DOM (mDOM) is an optical module with 24 3-inch photo-multiplayers (PMTs). This type of DOM will have 2.2 times higher photocathode area in comparison with IceCube pDOM, isotropic sensitivity, and opportunity to obtain information from individual "pixels" (24 PMTs). To be able to use already developed and well-tested techniques to reconstruct simulated events with new sensors, mDOMs were simulated as pDOM type modules, installed on existing IceCube strings, that have $\sim 3$ times higher quantum efficiency and isotropic angular acceptance.

The IceCube-Gen2 point source sensitivity is dominated by the detection and reconstruction capabilities for tracks produced in $v_{\mu} \mathrm{CC}$ interactions due to larger effective area and better angular resolution compared to cascades. Therefore, for this study, an isotropic distribution of muons having a power-law energy spectrum with a spectral index $\gamma=-1.4$ in the energy range between $E_{\text {min }}$ $=300 \mathrm{GeV}$ and $E_{\max }=100 \mathrm{PeV}$ is generated. The spectral index is chosen to keep the spectrum approximately flat in logarithmic scale after the event selection. Individual stochastic muon energy deposits are simulated inside of a cylindrical region that encloses IceCube-Gen2 with the height $H=1700 \mathrm{~m}$ and the radius $R=2500 \mathrm{~m}$. In the next step, the CLSim package is used - a simulation software package to propagate photons from Cherenkov radiation [6]. The final simulation step is the detector simulation that simulates noise and the charge response of the PMTs.

The properties of the simulated tracks are reconstructed using IceCube's reconstruction algorithms. The best performing muon track direction reconstruction algorithm (SplineMPE) is based on a maximum likelihood method using the arrival time distribution of Cherenkov photons registered by the experiment's PMTs [7]. It uses the results of simpler reconstruction algorithms, such as the LineFit algorithm [8], as starting hypothesis.

Eight datasets (one per geometry) were produced containing $\sim 50000$ triggered events in each. 

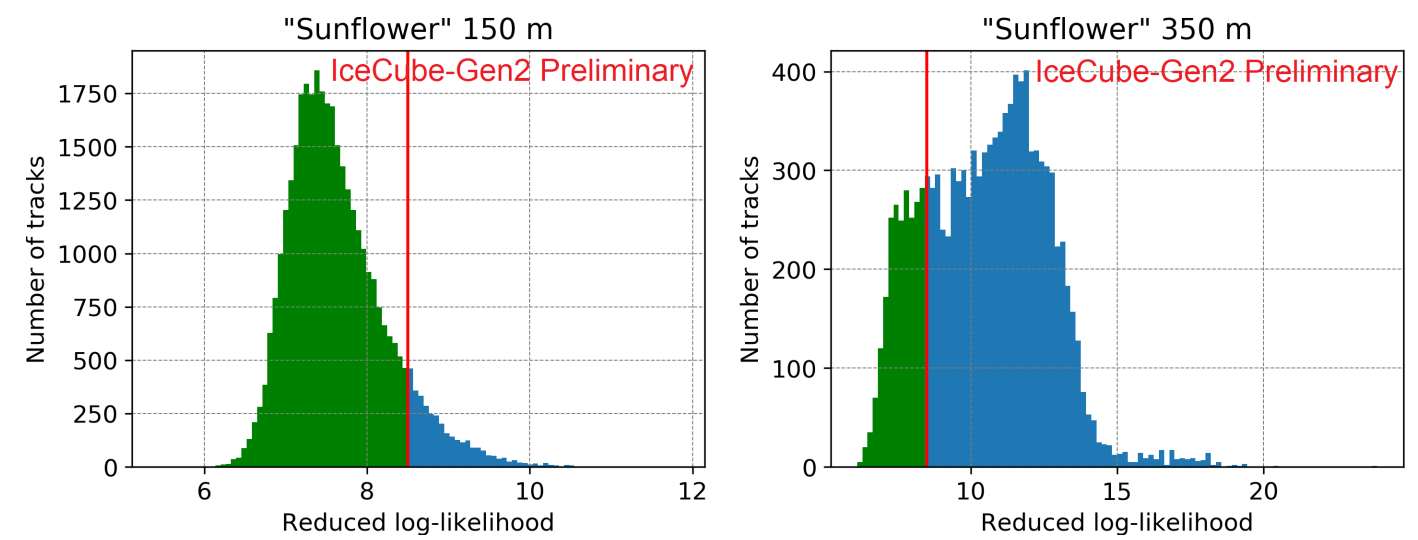

Figure 2: Distribution of the reduced log-likelihood parameter for two different high-energy array geometries. The part of dataset remaining when applying the (unaltered) cut motivated from IceCube analysis is marked green.

\section{Event selection}

An event selection is needed to exclude poorly reconstructed tracks from the dataset. Four parameters are used to discriminate well-reconstructed tracks from poorly-reconstructed tracks:

- LineFit velocity is a parameter describing an apparent track velocity in the LineFit algorithm. As a "sanity cut", this value should be less than twice the speed of light in ice. The major part of IceCube events have LineFit velocity less than speed of light in ice.

- Reduced log-likelihood is the absolute value of the best-fit log-likelihood divided by the number of degrees of freedom which is analogous to $\chi^{2} / n_{\text {dof }}$. "Good" tracks in IceCube have a value of less than 8.5 in this variable.

- Number of DOMs with "direct" photons, i.e. that arrive between $-15 \mathrm{~ns}$ and $+50 \mathrm{~ns}$ of the expected arrival time in a scatter-free medium, given the best-fit track. Good quality tracks in IceCube have a value larger than 6 for this parameter.

- Track length within the instrumented volume of IceCube. The direction of tracks that intersect only a small portion of the instrumented volume can usually not be well reconstructed. Therefore, the reconstructed track length must be greater than $120 \mathrm{~m}$ in IceCube.

The IceCube quality cuts might be inapplicable for IceCube-Gen2, therefore the performance of this event selection was examined for Gen2. The distributions of LineFit velocity, number of DOMs with direct photons, and track length did not change with inclusion of the high-energy array, unlike the reduced log-likelihood, which heavily depends on the spacing parameter. This is demonstrated also in Fig. 2: The part of dataset that remains after the standard IceCube selection procedure is marked green (on left from the red line). The high cut efficiency for IceCube (125 m inner-string spacing) or "Sunflower" $150 \mathrm{~m}$ drops dramatically for the Sunflower with $350 \mathrm{~m}$ interstring spacing. Therefore the reduced log-likelihood parameter had to be re-optimized individually for each geometry for this study. 


\begin{tabular}{|c|c|c|c|c|c|c|c|c|c|c|}
\hline \multirow{2}{*}{$\mathrm{s}, \mathrm{m}$} & \multirow{2}{*}{ 'rlogl' cut } & \multicolumn{3}{|c|}{ Zenith $90 \pm 10^{\circ}$} & \multicolumn{3}{|c|}{ Zenith $70 \pm 10^{\circ}$} & \multicolumn{3}{|c|}{ Zenith $50 \pm 10^{\circ}$} \\
\hline & & $\eta$ & $\Psi_{50}$ & $\Psi_{99}$ & $\eta$ & $\Psi_{50}$ & $\Psi_{99}$ & $\eta$ & $\Psi_{50}$ & $\Psi_{99}$ \\
\hline 150 & 8.5 & $97 \%$ & 0.23 & 3.8 & $93 \%$ & 0.27 & 10.9 & $90 \%$ & 0.28 & 14.3 \\
\hline 200 & 9.0 & $96 \%$ & 0.21 & 5.0 & $93 \%$ & 0.33 & 11.8 & $89 \%$ & 0.36 & 14.8 \\
\hline 220 & 9.0 & $97 \%$ & 0.22 & 6.1 & $97 \%$ & 0.33 & 11.2 & $93 \%$ & 0.40 & 31.4 \\
\hline 240 & 9.5 & $98 \%$ & 0.23 & 4.9 & $98 \%$ & 0.40 & 17.4 & $96 \%$ & 0.43 & 13.3 \\
\hline 260 & 9.5 & $95 \%$ & 0.22 & 8.5 & $94 \%$ & 0.30 & 10.3 & $89 \%$ & 0.41 & 18.9 \\
\hline 280 & 10.0 & $96 \%$ & 0.20 & 5.2 & $92 \%$ & 0.35 & 8.1 & $86 \%$ & 0.49 & 12.7 \\
\hline 300 & 10.5 & $93 \%$ & 0.20 & 8.3 & $89 \%$ & 0.31 & 8.2 & $87 \%$ & 0.49 & 18.7 \\
\hline 350 & 11.5 & $95 \%$ & 0.21 & 7.7 & $78 \%$ & 0.34 & 7.6 & $72 \%$ & 0.57 & 20.7 \\
\hline
\end{tabular}

Table 1: Reduced log-likelihood cuts optimized for the high-energy muon sample $\left(E_{\mu}>100 \mathrm{TeV}\right) . \eta$ denotes cut efficiency on this sample (i.e. fraction of events that remain after the application of the cut), $\Psi_{50}$ is the median opening angle (degrees), and $\Psi_{99}$ is the $99 \%$ quantile of the opening angle (degrees).

Two alternate sets of cuts were defined monitoring the selection efficiency in two different energy ranges. A high-energy (HE) set, aiming for a high efficiency for muons with $E_{\mu}>100 \mathrm{TeV}$, and a low-energy (LE) set, aiming for a high-efficiency for muons with $E_{\mu}<50 \mathrm{TeV}$. As the former selection is more stringent than the latter, the LE selection implicitly features a high efficiency also for high-energy tracks. The efficiency is checked separately in three zenith bins. The main criterion for defining the cut values was to maintain a small median opening angle between true and reconstructed direction and have simultaneously a good cut efficiency. In addition, the $99 \%$ quantile of the point spread function was considered as an estimate for the size of the tails of poorly reconstructed events in the set. Lacking a dedicated simulation of atmospheric muon background, the $99 \%$ quantile of the PSF has been used as a proxy for the angular reconstruction performance on this background. The selection cut has been adjusted to ensure that $\geq 99 \%$ of the muons arriving from zenith angles $\leq 80$ degrees are reconstructed outside the region with zenith angles $\geq 90^{\circ}$. The values of the selection cuts, as well as median opening angle, 99\% quantile for the point spread function (PSF), and cut efficiency are shown in Table 1 and Table 2 for the high- and low-energy sample, respectively. For horizontal muons, the angular resolution is approximately the same for all geometries. However, in zenith bin $50 \pm 10^{\circ}$, the denser instrumentation will show significantly better angular resolution.

\section{Sensitivity to astrophysical neutrinos}

In the following, we use the method described in [9] to obtain expected neutrino event rates and point source sensitivities from basic detector performance quantities such as the muon effective area and the energy resolution.

The performance of each of the eight proposed detector geometries was parametrized. For muon tracks, the detector performance is characterized by four quantities: the muon effective area, the selection efficiency, the energy resolution, and the point spread function.

Muon effective area. Fig. 3 shows the muon effective area of the "Sunflower" geometries with spacings from $150 \mathrm{~m}$ to $350 \mathrm{~m}$ for muons entering the detector with $1 \mathrm{PeV}$ energy. 


\begin{tabular}{|c|c|c|c|c|c|c|c|c|c|c|}
\hline \multirow{2}{*}{$\mathrm{s}, \mathrm{m}$} & \multirow{2}{*}{ 'rlogl' cut } & \multicolumn{3}{|c|}{ Zenith $90 \pm 10^{\circ}$} & \multicolumn{3}{|c|}{ Zenith $70 \pm 10^{\circ}$} & \multicolumn{3}{|c|}{ Zenith $50 \pm 10^{\circ}$} \\
\hline & & $\eta$ & $\Psi_{50}$ & $\Psi_{99}$ & $\eta$ & $\Psi_{50}$ & $\Psi_{99}$ & $\eta$ & $\Psi_{50}$ & $\Psi_{99}$ \\
\hline 150 & 8.5 & $91 \%$ & 0.24 & 5.4 & $90 \%$ & 0.34 & 6.9 & $87 \%$ & 0.44 & 10.8 \\
\hline 200 & 9.5 & $89 \%$ & 0.26 & 7.2 & $88 \%$ & 0.37 & 9.5 & $85 \%$ & 0.48 & 15.3 \\
\hline 220 & 10.0 & $88 \%$ & 0.25 & 6.9 & $88 \%$ & 0.36 & 10.1 & $85 \%$ & 0.51 & 12.9 \\
\hline 240 & 10 & $90 \%$ & 0.25 & 6.6 & $89 \%$ & 0.38 & 9.5 & $87 \%$ & 0.54 & 16.9 \\
\hline 260 & 10.5 & $83 \%$ & 0.25 & 9.2 & $82 \%$ & 0.38 & 13.9 & $81 \%$ & 0.55 & 15.2 \\
\hline 280 & 11.0 & $84 \%$ & 0.26 & 7.7 & $84 \%$ & 0.40 & 8.6 & $79 \%$ & 0.53 & 20.2 \\
\hline 300 & 11.5 & $84 \%$ & 0.26 & 8.5 & $84 \%$ & 0.40 & 13.0 & $78 \%$ & 0.59 & 15.8 \\
\hline 350 & 12.5 & $83 \%$ & 0.27 & 6.6 & $78 \%$ & 0.40 & 13.9 & $77 \%$ & 0.58 & 21.0 \\
\hline
\end{tabular}

Table 2: Reduced log-likelihood cuts optimized for the low-energy muon subsample $\left(E_{\mu}<50 \mathrm{TeV}\right) . \eta$ denotes cut efficiency on this sample (i.e. fraction of events that remain after the application of the cut), $\Psi_{50}$ is the median opening angle (degrees), and $\Psi_{99}$ is the $99 \%$ quantile of the opening angle (degrees).

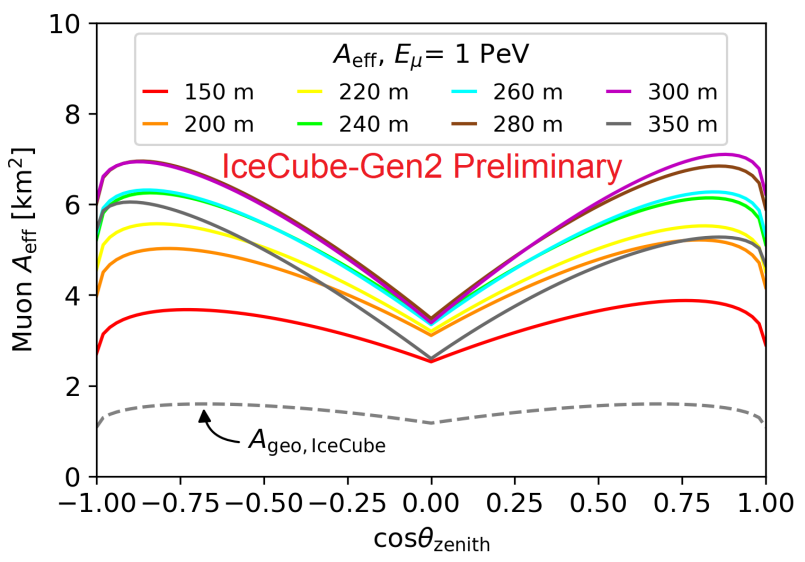

Figure 3: The average muon effective area after quality cuts as a function of zenith angle is shown for $1 \mathrm{PeV}$ muons.

Muon energy resolution. The stochastic energy losses of highly energetic muons cause a significant spread in observed muon energy. The muon energy resolution is a parameterization of the reconstructed energy distribution for muons entering the detector with a specific energy, after the selection described above.

Muon selection efficiency is a ratio of the number of tracks that passed selection cuts to the full number of triggered tracks.

Point spread function parameterizes the distribution of the angular distance between the true muon direction and the reconstructed direction as a function of zenith angle and muon energy.

\section{Point source discovery potential}

The $5 \sigma$ discovery potential for point sources is used as measure to compare the sensitivity of different inter-string spacings. It is defined as the neutrino flux from a candidate neutrino source that is required to produce an excess of neutrinos over background equivalent to a significance of $5 \sigma$ in half of all simulated experiments. 


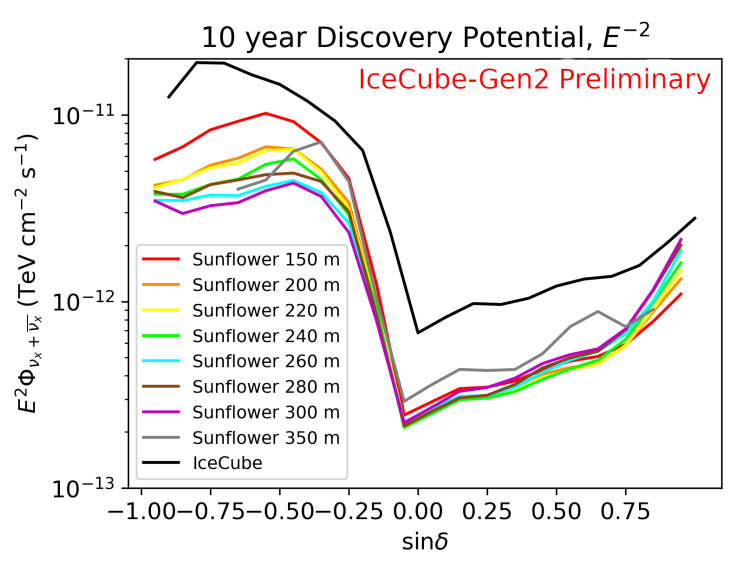

(a) HE cuts set for event selection

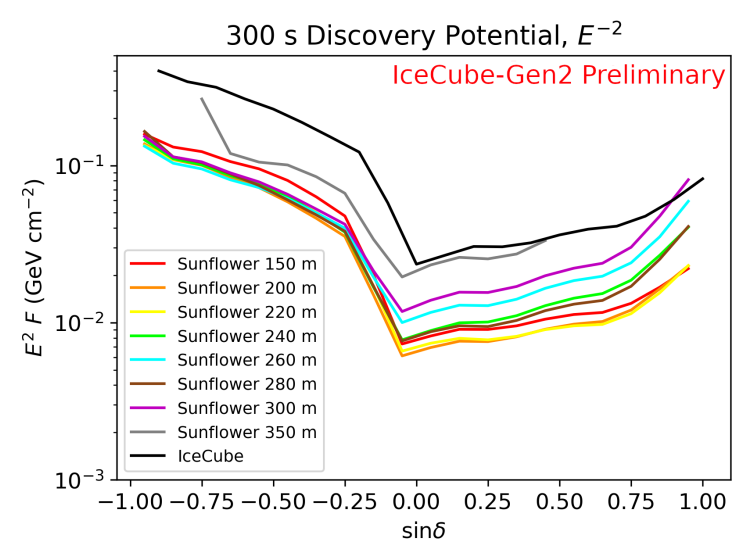

(b) LE cuts set event selection

Figure 4: IceCube-Gen2 (optical array, "Sunflower" geometries) discovery potential for (a) 10 years of exposure, high-energy cuts applied, (b) 300 seconds of exposure, low-energy cuts applied.

While IceCube measurements have confirmed the existence of an astrophysical neutrino flux in multiple energy bands and for different neutrino flavors [10-12], the observation of individual sources is limited to only few candidates [13, 14]. However, the significantly larger instrumented area of IceCube-Gen2 will allow detection of fluxes from individual sources that are much fainter than current limits.

Figures 4 (a) and 4 (b) show the discovery potentials to an $E^{-2}$ flux from a single source over 10 years and 300 seconds of exposure time, respectively, using only through-going tracks made by muons formed around IceCube and passing through the detector. The atmospheric background is assumed to be isotropic within one declination bin. For long exposure, the values of discovery potentials for the geometries with inter-spacing parameters from $200 \mathrm{~m}$ to $300 \mathrm{~m}$ differ only slightly (especially at the horizon where these values coincide within 5\% margin). "Sunflower" $350 \mathrm{~m}$ and "Sunflower" $150 \mathrm{~m}$ would have slightly worse sensitivity at the horizon compared to other geometries. For the "burst" discovery potentials (short signal duration), the smaller spacing values lead to better discovery potential, although the difference between the values of discovery potential is relatively small, as in case of 10 years of exposure.

The sensitivity in the direction of the Northern celestial pole is lower, because a significant fraction of high-energy neutrinos is absorbed in the Earth. In the Southern sky, the overall sensitivity is reduced due to the energy threshold imposed by the rejection of CR muon background, and the limited target mass for neutrino interactions between the surface and the detector. The largest projected density of instrumentation, the available target material and the large possible range of neutrino-induced muons make the celestial horizon $(\delta=0)$ the region with the best sensitivity for both, IceCube and IceCube-Gen2.

\section{Summary}

IceCube-Gen2 will be a multi-component facility that targets the detection of neutrinos with energies from several $\mathrm{GeV}$ to several $\mathrm{EeV}$. Here we have presented sensitivity studies for the highenergy optical array by varying the inter-string spacing. Good quality tracks have been selected 
to determine the relation between point source discovery potential and inter-string spacing. Two selections have been defined, one that focuses on high efficiency for tracks with energies below $50 \mathrm{TeV}$ and one that focuses on high efficiency for tracks above $100 \mathrm{TeV}$. For sources with a generic power-law spectrum with index -2, inter-string spacings between $200 \mathrm{~m}$ and $280 \mathrm{~m}$ show a similar performance with both event selections, while for larger and smaller inter-string spacings the discovery potential is comparatively worse.

\section{References}

[1] IceCube Collaboration, M. G. Aartsen et al. JINST 12 no. 03, (2017) P03012.

[2] IceCube-Gen2 Collaboration, M. G. Aartsen et al. Journal of Physics G: Nuclear and Particle Physics 48 no. 6, (Apr, 2021) 060501.

[3] ARA Collaboration, P. Allison, J. Auffenberg, R. Bard, J. Beatty, D. Besson, C. Bora, C.-C. Chen, P. Chen, A. Connolly, J. Davies, and et al. Astroparticle Physics 70 (Oct, 2015) 62-80.

[4] IceCube Collaboration, A. Ishihara PoS ICRC2019 (2019) 1031.

[5] J. Lünemann, P. Coppin, and S. Toscano, "In-ice self-veto techniques for IceCube-Gen2," in Proceedings of 35th International Cosmic Ray Conference - PoS(ICRC2017), vol. 301, p. 945.2017.

[6] D. Chirkin, J. C. Díaz-Vélez, C. Kopper, A. Olivas, B. Riedel, M. Rongen, D. Schultz, and J. van Santen, "Photon propagation using gpus by the icecube neutrino observatory," in 2019 15th International Conference on eScience (eScience), pp. 388-393. 2019.

[7] IceCube Collaboration, R. Abbasi et al. arXiv 2103.16931 .

[8] V. J. Stenger, Track Fitting For Dumand-II Octagon Array (technical report). University of Hawaii at Manoa, 1990.

[9] IceCube-Gen2 Collaboration, J. van Santen PoS ICRC2017 (2018) 991.

[10] IceCube Collaboration, H. M. Niederhausen and Y. Xu PoS ICRC2017 (2018) 968.

[11] IceCube Collaboration, R. Abbasi et al. arXiv 2011.03545 .

[12] IceCube Collaboration, J. Stettner PoS ICRC2019 (2019) 1017.

[13] M. Aartsen, M. Ackermann, J. Adams, J. A. Aguilar, M. Ahlers, M. Ahrens, I. Al Samarai, D. Altmann, K. Andeen, and et al. Science 361 no. 6398, (Jul, 2018) 147-151.

[14] R. Stein, S. v. Velzen, M. Kowalski, A. Franckowiak, S. Gezari, J. C. A. Miller-Jones, S. Frederick, I. Sfaradi, M. F. Bietenholz, A. Horesh, and et al. Nature Astronomy 5 no. 5, (Feb, 2021) 510-518. 


\section{Full Author List: IceCube-Gen2 Collaboration}

R. Abbasi ${ }^{17}$, M. Ackermann ${ }^{71}$, J. Adams ${ }^{22}$, J. A. Aguilar ${ }^{12}$, M. Ahlers ${ }^{26}$, M. Ahrens ${ }^{60}$, C. Alispach ${ }^{32}$, P. Allison ${ }^{24,25}$, A. A. Alves $\mathrm{Jr}^{35}$, N. M. Amin ${ }^{50}$, R. An ${ }^{14}$, K. Andeen ${ }^{48}$, T. Anderson ${ }^{67}$, G. Anton ${ }^{30}$, C. Argüelles ${ }^{14}$, T. C. Arlen ${ }^{67}$, Y. Ashida ${ }^{45}$, S. Axani ${ }^{15}, \mathrm{X}$. Bai $^{56}$, A. Balagopal V. ${ }^{45}$, A. Barbano ${ }^{32}$, I. Bartos ${ }^{52}$, S. W. Barwick ${ }^{34}$, B. Bastian ${ }^{71}$, V. Basu ${ }^{45}$, S. Baur ${ }^{12}$, R. Bay ${ }^{8}$, J. J. Beatty ${ }^{24,}$ 25, $^{25}$, K.-H. Becker ${ }^{70}$, J. Becker Tjus ${ }^{11}$, C. Bellenghi ${ }^{31}$, S. BenZvi ${ }^{58}$, D. Berley ${ }^{23}$, E. Bernardini ${ }^{71}$, 72, D. Z. Besson ${ }^{38,73}$, G. Binder ${ }^{8,9}$, D. Bindig $^{70}$, A. Bishop ${ }^{45}$, E. Blaufuss ${ }^{23}$, S. Blot ${ }^{71}$, M. Boddenberg ${ }^{1}$, M. Bohmer ${ }^{31}$, F. Bontempo ${ }^{35}$, J. Borowka ${ }^{1}$, S. Böser ${ }^{46}$, O. Botner ${ }^{69}$, J. Böttcher ${ }^{1}$, E. Bourbeau ${ }^{26}$, F. Bradascio ${ }^{71}$, J. Braun ${ }^{45}$, S. Bron ${ }^{32}$, J. Brostean-Kaiser ${ }^{71}$, S. Browne ${ }^{36}$, A. Burgman ${ }^{69}$, R. T. Burley ${ }^{2}$, R. S. Busse ${ }^{49}$, M. A. Campana ${ }^{55}$, E. G. Carnie-Bronca ${ }^{2}$, M. Cataldo ${ }^{30}$, C. Chen ${ }^{6}$, D. Chirkin ${ }^{45}$, K. Choi ${ }^{62}$, B. A. Clark ${ }^{28}$, K. Clark ${ }^{37}$, R. Clark $^{40}$, L. Classen ${ }^{49}$, A. Coleman ${ }^{50}$, G. H. Collin ${ }^{15}$, A. Connolly ${ }^{24}$, 25 , J. M. Conrad ${ }^{15}$, P. Coppin ${ }^{13}$, P. Correa ${ }^{13}$, D. F. Cowen ${ }^{66,67}$, R. $\mathrm{Cross}^{58}$, C. Dappen ${ }^{1}$, P. Dave ${ }^{6}$, C. Deaconu ${ }^{20,21}$, C. De Clercq ${ }^{13}$, S. De Kockere ${ }^{13}$, J. J. DeLaunay ${ }^{67}$, H. Dembinski ${ }^{50}$, K. Deoskar ${ }^{60}$, S. De Ridder ${ }^{33}$, A. Desai ${ }^{45}$, P. Desiati ${ }^{45}$, K. D. de Vries ${ }^{13}$, G. de Wasseige ${ }^{13}$, M. de With ${ }^{10}$, T. DeYoung ${ }^{28}$, S. Dharani ${ }^{1}$, A. Diaz $^{15}$, J. C. Díaz-Vélez ${ }^{45}$, M. Dittmer ${ }^{49}$, H. Dujmovic ${ }^{35}$, M. Dunkman ${ }^{67}$, M. A. DuVernois ${ }^{45}$, E. Dvorak ${ }^{56}$, T. Ehrhardt ${ }^{46}$, P. Eller ${ }^{31}$, R. Engel $^{35,36}$, H. Erpenbeck ${ }^{1}$, J. Evans ${ }^{23}$, J. J. Evans ${ }^{47}$, P. A. Evenson ${ }^{50}$, K. L. Fan ${ }^{23}$, K. Farrag ${ }^{41}$, A. R. Fazely ${ }^{7}$, S. Fiedlschuster ${ }^{30}$, A. T. Fienberg ${ }^{67}$, K. Filimonov ${ }^{8}$, C. Finley ${ }^{60}$, L. Fischer ${ }^{71}$, D. Fox ${ }^{66}$, A. Franckowiak ${ }^{11,71}$, E. Friedman ${ }^{23}$, A. Fritz ${ }^{46}$, P. Fürst ${ }^{1}$, T. K. Gaisser ${ }^{50}$, J. Gallagher ${ }^{44}$, E. Ganster ${ }^{1}$, A. Garcia ${ }^{14}$, S. Garrappa ${ }^{71}$, A. Gartner ${ }^{31}$, L. Gerhardt ${ }^{9}$, R. Gernhaeuser ${ }^{31}$, A. Ghadimi ${ }^{65}$, P. Giri ${ }^{39}$, C. Glaser ${ }^{69}$, T. Glauch ${ }^{31}$, T. Glüsenkamp ${ }^{30}$, A. Goldschmidt ${ }^{9}$, J. G. Gonzalez ${ }^{50}$, S. Goswami ${ }^{65}$, D. Grant ${ }^{28}$, T. Grégoire ${ }^{67}$, S. Griswold ${ }^{58}$, M. Gündüz ${ }^{11}$, C. Günther ${ }^{1}$, C. Haack ${ }^{31}$, A. Hallgren ${ }^{69}$, R. Halliday ${ }^{28}$, S. Hallmann ${ }^{71}$, L. Halve ${ }^{1}$, F. Halzen ${ }^{45}$, M. $\mathrm{Ha} \mathrm{Minh}^{31}$, K. Hanson ${ }^{45}$, J. Hardin ${ }^{45}$, A. A. Harnisch ${ }^{28}$, J. Haugen ${ }^{45}$, A. Haungs ${ }^{35}$, S. Hauser ${ }^{1}$, D. Hebecker ${ }^{10}$, D. Heinen ${ }^{1}$, K.

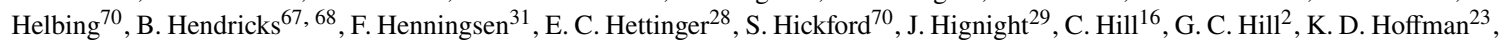
B. Hoffmann ${ }^{35}$, R. Hoffmann ${ }^{70}$, T. Hoinka ${ }^{27}$, B. Hokanson-Fasig ${ }^{45}$, K. Holzapfel ${ }^{31}$, K. Hoshina ${ }^{45,64}$, F. Huang ${ }^{67}$, M. Huber ${ }^{31}$, T.

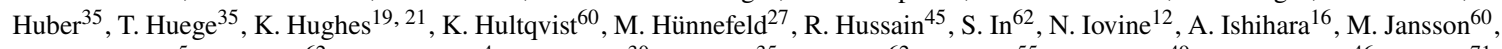
G. S. Japaridze ${ }^{5}$, M. Jeong ${ }^{62}$, B. J. P. Jones ${ }^{4}$, O. Kalekin ${ }^{30}$, D. Kang ${ }^{35}$, W. Kang ${ }^{62}$, X. Kang ${ }^{55}$, A. Kappes ${ }^{49}$, D. Kappesser ${ }^{46}$, T. Karg ${ }^{71}$, M. Karl ${ }^{31}$, A. Karle ${ }^{45}$, T. Katori ${ }^{40}$, U. Katz ${ }^{30}$, M. Kauer ${ }^{45}$, A. Keivani ${ }^{52}$, M. Kellermann ${ }^{1}$, J. L. Kelley ${ }^{45}$, A. Kheirandish ${ }^{67}$, K. Kin ${ }^{16}$, T. Kintscher ${ }^{71}$, J. Kiryluk ${ }^{61}$, S. R. Klein ${ }^{8,9}$, R. Koirala ${ }^{50}$, H. Kolanoski ${ }^{10}$, T. Kontrimas ${ }^{31}$, L. Köpke ${ }^{46}$, C. Kopper ${ }^{28}$, S. Kopper ${ }^{65}$, D. J. Koskinen ${ }^{26}$, P. Koundal ${ }^{35}$, M. Kovacevich ${ }^{55}$, M. Kowalski ${ }^{10,71}$, T. Kozynets ${ }^{26}$, C. B. Krauss ${ }^{29}$, I. Kravchenko ${ }^{39}$, R. Krebs ${ }^{67}{ }^{68}$, E.

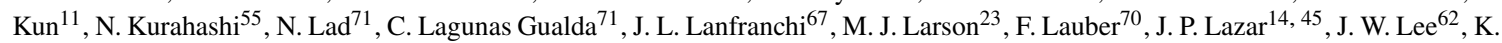

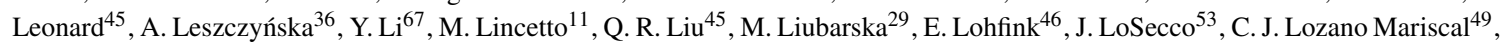
L. $\mathrm{Lu}^{45}$, F. Lucarelli ${ }^{32}$, A. Ludwig ${ }^{28,42}$, W. Luszczak ${ }^{45}$, Y. Lyu ${ }^{8,9}$, W. Y. Ma ${ }^{71}$, J. Madsen ${ }^{45}$, K. B. M. Mahn ${ }^{28}$, Y. Makino ${ }^{45}$, S. Mancina $^{45}$, S. Mandalia ${ }^{41}$, I. C. Mariş ${ }^{12}$, S. Marka ${ }^{52}$, Z. Marka ${ }^{52}$, R. Maruyama ${ }^{51}$, K. Mase ${ }^{16}$, T. McElroy ${ }^{29}$, F. McNally ${ }^{43}$, J. V. Mead $^{26}$, K. Meagher ${ }^{45}$, A. Medina ${ }^{25}$, M. Meier ${ }^{16}$, S. Meighen-Berger ${ }^{31}$, Z. Meyers ${ }^{71}$, J. Micallef ${ }^{28}$, D. Mocklerr ${ }^{12}$, T. Montaruli ${ }^{32}$, R. W. Moore ${ }^{29}$, R. Morse ${ }^{45}$, M. Moulai ${ }^{15}$, R. Naab ${ }^{71}$, R. Nagai ${ }^{16}$, U. Naumann ${ }^{70}$, J. Necker ${ }^{71}$, A. Nelles ${ }^{30,71}$, L. V. Nguyễn ${ }^{28}$, H. Niederhausen $^{31}$, M. U. Nisa ${ }^{28}$, S. C. Nowicki ${ }^{28}$, D. R. Nygren ${ }^{9}$, E. Oberla ${ }^{20,21}$, A. Obertacke Pollmann ${ }^{70}$, M. Oehler ${ }^{35}$, A. Olivas ${ }^{23}$, A. Omeliukh ${ }^{71}$, E. O' $\operatorname{Sullivan}^{69}$, H. Pandya ${ }^{50}$, D. V. Pankova ${ }^{67}$, L. Papp ${ }^{31}$, N. Park ${ }^{37}$, G. K. Parker ${ }^{4}$, E. N. Paudel ${ }^{50}$, L. Paul ${ }^{48}$, C. Pérez de $\operatorname{los} \operatorname{Heros}^{69}$, L. Peters ${ }^{1}$, T. C. Petersen ${ }^{26}$, J. Peterson ${ }^{45}$, S. Philippen ${ }^{1}$, D. Pieloth ${ }^{27}$, S. Pieper ${ }^{70}$, J. L. Pinfold ${ }^{29}$, M. Pittermann ${ }^{36}$, A. Pizzuto $^{45}$, I. Plaisier ${ }^{71}$, M. Plum ${ }^{48}$, Y. Popovych ${ }^{46}$, A. Porcelli ${ }^{33}$, M. Prado Rodriguez ${ }^{45}$, P. B. Price ${ }^{8}$, B. Pries ${ }^{28}$, G. T. Przybylski ${ }^{9}$, L. Pyras $^{71}$, C. Raab ${ }^{12}$, A. Raissi ${ }^{22}$, M. Rameez ${ }^{26}$, K. Rawlins ${ }^{3}$, I. C. Rea ${ }^{31}$, A. Rehman ${ }^{50}$, P. Reichherzer ${ }^{11}$, R. Reimann ${ }^{1}$, G. Renzi ${ }^{12}$, E. Resconi ${ }^{31}$, S. Reusch ${ }^{71}$, W. Rhode 27 , M. Richman ${ }^{55}$, B. Riedel ${ }^{45}$, M. Riegel ${ }^{35}$, E. J. Roberts ${ }^{2}$, S. Robertson ${ }^{8,9}$, G. Roellinghoff ${ }^{62}$, M. Rongen ${ }^{46}$, C. Rott ${ }^{59}$ 62, T. Ruhe ${ }^{27}$, D. Ryckbosch ${ }^{33}$, D. Rysewyk Cantu ${ }^{28}$, I. Safa ${ }^{14,45}$, J. Saffer ${ }^{36}$, S. E. Sanchez Herrera ${ }^{28}$, A. Sandrock $^{27}$, J. Sandroos ${ }^{46}$, P. Sandstrom ${ }^{45}$, M. Santander ${ }^{65}$, S. Sarkar ${ }^{54}$, S. Sarkar ${ }^{29}$, K. Satalecka ${ }^{71}$, M. Scharf ${ }^{1}$, M. Schaufel ${ }^{1}$, H. Schieler $^{35}$, S. Schindler ${ }^{30}$, P. Schlunder ${ }^{27}$, T. Schmidt ${ }^{23}$, A. Schneider ${ }^{45}$, J. Schneider ${ }^{30}$, F. G. Schröder ${ }^{35}$, 50, L. Schumacher ${ }^{31}$, G. Schwefer $^{1}$, S. Sclafani ${ }^{55}$, D. Seckel ${ }^{50}$, S. Seunarine ${ }^{57}$, M. H. Shaevitz ${ }^{52}$, A. Sharma ${ }^{69}$, S. Shefali ${ }^{36}$, M. Silva ${ }^{45}$, B. Skrzypek ${ }^{14}$, D. Smith $^{19,21}$, B. Smithers ${ }^{4}$, R. Snihur ${ }^{45}$, J. Soedingrekso ${ }^{27}$, D. Soldin ${ }^{50}$, S. Söldner-Rembold ${ }^{47}$, D. Southall ${ }^{19,21}$, C. Spannfellner ${ }^{31}$, G. M. Spiczak ${ }^{57}$, C. Spiering ${ }^{71}$, 73, J. Stachurska ${ }^{71}$, M. Stamatikos ${ }^{25}$, T. Stanev ${ }^{50}$, R. Stein ${ }^{71}$, J. Stettner ${ }^{1}$, A. Steuer ${ }^{46}$, T. Stezelberger ${ }^{9}$, T.

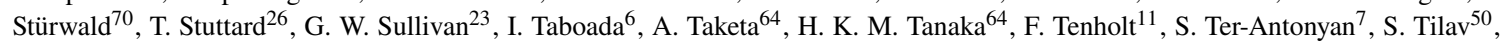
F. Tischbein ${ }^{1}$, K. Tollefson ${ }^{28}$, L. Tomankova ${ }^{11}$, C. Tönnis ${ }^{63}$, J. Torres ${ }^{24,25}$, S. Toscano ${ }^{12}$, D. Tosi ${ }^{45}$, A. Trettin ${ }^{71}$, M. Tselengidou ${ }^{30}$, C. F. Tung ${ }^{6}$, A. Turcati $^{31}$, R. Turcotte ${ }^{35}$, C. F. Turley ${ }^{67}$, J. P. Twagirayezu ${ }^{28}$, B. Ty ${ }^{45}$, M. A. Unland Elorrieta ${ }^{49}$, N. Valtonen-Mattila ${ }^{69}$, J. Vandenbroucke ${ }^{45}$, N. van Eijndhoven ${ }^{13}$, D. Vannerom ${ }^{15}$, J. van Santen $^{71}$, D. Veberic ${ }^{35}$, S. Verpoest ${ }^{33}$, A. Vieregg ${ }^{18,}$ 19, 20, 21, M. Vraeghe $^{33}$, C. Walck ${ }^{60}$, T. B. Watson ${ }^{4}$, C. Weaver ${ }^{28}$, P. Weigel ${ }^{15}$, A. Weindl ${ }^{35}$, L. Weinstock ${ }^{1}$, M. J. Weiss ${ }^{67}$, J. Weldert ${ }^{46}$, C. Welling ${ }^{71}$, C. Wendt ${ }^{45}$, J. Werthebach ${ }^{27}$, M. Weyrauch ${ }^{36}$, N. Whitehorn ${ }^{28,}{ }^{42}$, C. H. Wiebusch ${ }^{1}$, D. R. Williams ${ }^{65}$, S. Wissel ${ }^{66,67,68}$, M. Wolf $^{31}$, K. Woschnagg ${ }^{8}$, G. Wrede ${ }^{30}$, S. Wren ${ }^{47}$, J. Wulff ${ }^{11}$, X. W. Xu ${ }^{7}$, Y. Xu ${ }^{61}$, J. P. Yanez ${ }^{29}$, S. Yoshida ${ }^{16}$, S. Yu ${ }^{28}$, T. Yuan ${ }^{45}$, Z. Zhang ${ }^{61}$, S. Zierke ${ }^{1}$

\footnotetext{
${ }^{1}$ III. Physikalisches Institut, RWTH Aachen University, D-52056 Aachen, Germany

${ }^{2}$ Department of Physics, University of Adelaide, Adelaide, 5005, Australia

${ }^{3}$ Dept. of Physics and Astronomy, University of Alaska Anchorage, 3211 Providence Dr., Anchorage, AK 99508, USA

${ }^{4}$ Dept. of Physics, University of Texas at Arlington, 502 Yates St., Science Hall Rm 108, Box 19059, Arlington, TX 76019, USA

${ }^{5}$ CTSPS, Clark-Atlanta University, Atlanta, GA 30314, USA

${ }^{6}$ School of Physics and Center for Relativistic Astrophysics, Georgia Institute of Technology, Atlanta, GA 30332, USA

${ }^{7}$ Dept. of Physics, Southern University, Baton Rouge, LA 70813, USA

${ }^{8}$ Dept. of Physics, University of California, Berkeley, CA 94720, USA
} 
${ }^{9}$ Lawrence Berkeley National Laboratory, Berkeley, CA 94720, USA

${ }^{10}$ Institut für Physik, Humboldt-Universität zu Berlin, D-12489 Berlin, Germany

${ }^{11}$ Fakultät für Physik \& Astronomie, Ruhr-Universität Bochum, D-44780 Bochum, Germany

${ }^{12}$ Université Libre de Bruxelles, Science Faculty CP230, B-1050 Brussels, Belgium

${ }^{13}$ Vrije Universiteit Brussel (VUB), Dienst ELEM, B-1050 Brussels, Belgium

${ }^{14}$ Department of Physics and Laboratory for Particle Physics and Cosmology, Harvard University, Cambridge, MA 02138, USA

${ }^{15}$ Dept. of Physics, Massachusetts Institute of Technology, Cambridge, MA 02139, USA

${ }^{16}$ Dept. of Physics and Institute for Global Prominent Research, Chiba University, Chiba 263-8522, Japan

${ }^{17}$ Department of Physics, Loyola University Chicago, Chicago, IL 60660, USA

${ }^{18}$ Dept. of Astronomy and Astrophysics, University of Chicago, Chicago, IL 60637, USA

${ }^{19}$ Dept. of Physics, University of Chicago, Chicago, IL 60637, USA

${ }^{20}$ Enrico Fermi Institute, University of Chicago, Chicago, IL 60637, USA

${ }^{21}$ Kavli Institute for Cosmological Physics, University of Chicago, Chicago, IL 60637, USA

22 Dept. of Physics and Astronomy, University of Canterbury, Private Bag 4800, Christchurch, New Zealand

${ }^{23}$ Dept. of Physics, University of Maryland, College Park, MD 20742, USA

${ }^{24}$ Dept. of Astronomy, Ohio State University, Columbus, OH 43210, USA

${ }^{25}$ Dept. of Physics and Center for Cosmology and Astro-Particle Physics, Ohio State University, Columbus, OH 43210, USA

${ }^{26}$ Niels Bohr Institute, University of Copenhagen, DK-2100 Copenhagen, Denmark

${ }^{27}$ Dept. of Physics, TU Dortmund University, D-44221 Dortmund, Germany

${ }^{28}$ Dept. of Physics and Astronomy, Michigan State University, East Lansing, MI 48824, USA

${ }^{29}$ Dept. of Physics, University of Alberta, Edmonton, Alberta, Canada T6G 2E1

${ }^{30}$ Erlangen Centre for Astroparticle Physics, Friedrich-Alexander-Universität Erlangen-Nürnberg, D-91058 Erlangen, Germany

${ }^{31}$ Physik-department, Technische Universität München, D-85748 Garching, Germany

${ }^{32}$ Département de physique nucléaire et corpusculaire, Université de Genève, CH-1211 Genève, Switzerland

${ }^{33}$ Dept. of Physics and Astronomy, University of Gent, B-9000 Gent, Belgium

${ }^{34}$ Dept. of Physics and Astronomy, University of California, Irvine, CA 92697, USA

${ }^{35}$ Karlsruhe Institute of Technology, Institute for Astroparticle Physics, D-76021 Karlsruhe, Germany

${ }^{36}$ Karlsruhe Institute of Technology, Institute of Experimental Particle Physics, D-76021 Karlsruhe, Germany

${ }^{37}$ Dept. of Physics, Engineering Physics, and Astronomy, Queen's University, Kingston, ON K7L 3N6, Canada

${ }^{38}$ Dept. of Physics and Astronomy, University of Kansas, Lawrence, KS 66045, USA

${ }^{39}$ Dept. of Physics and Astronomy, University of Nebraska-Lincoln, Lincoln, Nebraska 68588, USA

${ }^{40}$ Dept. of Physics, King's College London, London WC2R 2LS, United Kingdom

${ }^{41}$ School of Physics and Astronomy, Queen Mary University of London, London E1 4NS, United Kingdom

42 Department of Physics and Astronomy, UCLA, Los Angeles, CA 90095, USA

${ }^{43}$ Department of Physics, Mercer University, Macon, GA 31207-0001, USA

${ }^{44}$ Dept. of Astronomy, University of Wisconsin-Madison, Madison, WI 53706, USA

${ }^{45}$ Dept. of Physics and Wisconsin IceCube Particle Astrophysics Center, University of Wisconsin-Madison, Madison, WI 53706, USA

${ }^{46}$ Institute of Physics, University of Mainz, Staudinger Weg 7, D-55099 Mainz, Germany

${ }^{47}$ School of Physics and Astronomy, The University of Manchester, Oxford Road, Manchester, M13 9PL, United Kingdom

${ }^{48}$ Department of Physics, Marquette University, Milwaukee, WI, 53201, USA

${ }^{49}$ Institut für Kernphysik, Westfälische Wilhelms-Universität Münster, D-48149 Münster, Germany

${ }^{50}$ Bartol Research Institute and Dept. of Physics and Astronomy, University of Delaware, Newark, DE 19716, USA

${ }^{51}$ Dept. of Physics, Yale University, New Haven, CT 06520, USA

${ }^{52}$ Columbia Astrophysics and Nevis Laboratories, Columbia University, New York, NY 10027, USA

${ }^{53}$ Dept. of Physics, University of Notre Dame du Lac, 225 Nieuwland Science Hall, Notre Dame, IN 46556-5670, USA

${ }^{54}$ Dept. of Physics, University of Oxford, Parks Road, Oxford OX1 3PU, UK

${ }^{55}$ Dept. of Physics, Drexel University, 3141 Chestnut Street, Philadelphia, PA 19104, USA

${ }^{56}$ Physics Department, South Dakota School of Mines and Technology, Rapid City, SD 57701, USA

${ }^{57}$ Dept. of Physics, University of Wisconsin, River Falls, WI 54022, USA

${ }^{58}$ Dept. of Physics and Astronomy, University of Rochester, Rochester, NY 14627, USA

${ }^{59}$ Department of Physics and Astronomy, University of Utah, Salt Lake City, UT 84112, USA

${ }^{60}$ Oskar Klein Centre and Dept. of Physics, Stockholm University, SE-10691 Stockholm, Sweden

${ }^{61}$ Dept. of Physics and Astronomy, Stony Brook University, Stony Brook, NY 11794-3800, USA

62 Dept. of Physics, Sungkyunkwan University, Suwon 16419, Korea

${ }^{63}$ Institute of Basic Science, Sungkyunkwan University, Suwon 16419, Korea

${ }^{64}$ Earthquake Research Institute, University of Tokyo, Bunkyo, Tokyo 113-0032, Japan

${ }^{65}$ Dept. of Physics and Astronomy, University of Alabama, Tuscaloosa, AL 35487, USA

${ }^{66}$ Dept. of Astronomy and Astrophysics, Pennsylvania State University, University Park, PA 16802, USA

${ }^{67}$ Dept. of Physics, Pennsylvania State University, University Park, PA 16802, USA

${ }^{68}$ Institute of Gravitation and the Cosmos, Center for Multi-Messenger Astrophysics, Pennsylvania State University, University Park, PA 16802, USA 
${ }^{69}$ Dept. of Physics and Astronomy, Uppsala University, Box 516, S-75120 Uppsala, Sweden

${ }^{70}$ Dept. of Physics, University of Wuppertal, D-42119 Wuppertal, Germany

${ }^{71}$ DESY, D-15738 Zeuthen, Germany

72 Università di Padova, I-35131 Padova, Italy

${ }^{73}$ National Research Nuclear University, Moscow Engineering Physics Institute (MEPhI), Moscow 115409, Russia

\section{Acknowledgements}

USA - U.S. National Science Foundation-Office of Polar Programs, U.S. National Science Foundation-Physics Division, U.S. National Science Foundation-EPSCoR, Wisconsin Alumni Research Foundation, Center for High Throughput Computing (CHTC) at the University of Wisconsin-Madison, Open Science Grid (OSG), Extreme Science and Engineering Discovery Environment (XSEDE), Frontera computing project at the Texas Advanced Computing Center, U.S. Department of Energy-National Energy Research Scientific Computing Center, Particle astrophysics research computing center at the University of Maryland, Institute for Cyber-Enabled Research at Michigan State University, and Astroparticle physics computational facility at Marquette University; Belgium - Funds for Scientific Research (FRS-FNRS and FWO), FWO Odysseus and Big Science programmes, and Belgian Federal Science Policy Office (Belspo); Germany - Bundesministerium für Bildung und Forschung (BMBF), Deutsche Forschungsgemeinschaft (DFG), Helmholtz Alliance for Astroparticle Physics (HAP), Initiative and Networking Fund of the Helmholtz Association, Deutsches Elektronen Synchrotron (DESY), and High Performance Computing cluster of the RWTH Aachen; Sweden - Swedish Research Council, Swedish Polar Research Secretariat, Swedish National Infrastructure for Computing (SNIC), and Knut and Alice Wallenberg Foundation; Australia - Australian Research Council; Canada - Natural Sciences and Engineering Research Council of Canada, Calcul Québec, Compute Ontario, Canada Foundation for Innovation, WestGrid, and Compute Canada; Denmark - Villum Fonden and Carlsberg Foundation; New Zealand Marsden Fund; Japan - Japan Society for Promotion of Science (JSPS) and Institute for Global Prominent Research (IGPR) of Chiba University; Korea - National Research Foundation of Korea (NRF); Switzerland - Swiss National Science Foundation (SNSF); United Kingdom - Department of Physics, University of Oxford. 